Relations industrielles

Industrial Relations

\title{
Urban Land Use Planning. By F. Stuart Chapin Jr. New York: Harper \& Brothers, 1957. XV \& 397 p. \$8.00 (\$6.00 text book).
}

\section{Yves Martin}

Volume 13, numéro 1, janvier 1958

URI : https://id.erudit.org/iderudit/1022484ar

DOI : https://doi.org/10.7202/1022484ar

Aller au sommaire du numéro

Éditeur(s)

Département des relations industrielles de l'Université Laval

ISSN

0034-379X (imprimé)

1703-8138 (numérique)

Découvrir la revue

Citer ce compte rendu

Martin, Y. (1958). Compte rendu de [Urban Land Use Planning. By F. Stuart Chapin Jr. New York: Harper \& Brothers, 1957. XV \& 397 p. \$8.00 (\$6.00 text book).] Relations industrielles / Industrial Relations, 13(1), 115-116.

https://doi.org/10.7202/1022484ar

Tous droits réservés @ C Département des relations industrielles de l’Université Laval, 1958
Ce document est protégé par la loi sur le droit d'auteur. L'utilisation des services d'Érudit (y compris la reproduction) est assujettie à sa politique d'utilisation que vous pouvez consulter en ligne.

https://apropos.erudit.org/fr/usagers/politique-dutilisation/ 
Human Relations in Business, by Keith Davis, N.Y., McGraw-Hill, 1957, pp. 547.

Dans sa préface, l'auteur écrit: \&I have tried to present a book with content and substance rather than elementary platitudes and wheezes ». L'objectif est parfaitement atteint, car Human Relations in Business est du meilleur calibre en son genre. En plus d'ètre récent et à point, il est d'une clarté et d'une cohésion supérieures à la majorité des écrits sur le sujet. Les nombreux aspects des relations humaines sont analysés comme appartenant à un tout, et les différents points de vue sont exprimés avec objectivité, non dans un amas confus de matière mal digérée qui étourdit le lecteur, mais dans une discussion bien intégrée des fondements des relations humaines dans l'industrie. Le tout, abondamment supporté par des dessins, cartes, tableaux, graphiques et diagrammes qui illustrent les énoncés, par ane bibliographie complémentaire à la fin de chaque chapitre et, par l'étude d'une quinzaine de cas-problèmes choisis dans le monde industriel.

L'énumération de certains des points majeurs couverts donnera une idée de l'ampleur de la matière: L'entraînement, le «role playing », la participation dynamique des groupes, l'administration des salaires et des taux à la pièce, les méthodes de travail, «employee counseling », la négociation collective, le règlement des griefs et le moral.

Davis définit la nature des relations humaines, puis situe le problème au sein de l'organisation d'une entreprise. Dans sa partie maîtresse, il montre comment la direction peut améliorer ses relations avec ses employés à travers toutes les activités du personnel. Il ne demeure pas dans l'abstrait, mais aborde les problèmes avec l'optique que l'expérience a enseignée aux administrateurs. Il discute à la fois les méthodes directes et indirectes de promouvoir les relations de travail avec leurs faiblesses, leurs avantages et les difficultés qu'elles peuvent soulever.

L'auteur pose comme principe que les relations humaines sont un art plutôt qu'une science. Il aborde ainsi les rapports humains avec une conception humanitaire et sociale qui veut que les gens soient considérés comme des êtres humains. Keith Davis est un homme d'une grande compétence. Il est expert conseil pour de nombreuses entreprises et professeur en administration au School of Business, Indiana University. Ce volume est le fruit de vingt années d'expériences et de recherches dans l'industrie et l'enseignement. Il sera utile pour la formation du personnel de maîtrise, pour l'éducation des adultes et pour les écoles d'administration et gestion des entreprises.

\section{L.-M. Tremblay}

\section{Urban Land Use Planning. By F. Stuart} Chapin Jr. New York: Harper \& Brothers, 1957. XV \& 397 p. $\$ 8.00$ ( $\$ 6.00$ text book).

Les urbanistes affirment de plus en plus leur souci d'appuyer leur discipline, essentiellement tournée vers l'application, sur l'investigation scientifique, rigoureuse, de la réalité urbaine. A cette exigence des praticiens, le professeur Chapin tente, dans son ouvrage, d'apporter certains éléments de réponse. Son effort s'applique à un aspect fondamental de la planification urbaine: l'aménagement du cadre spatial de la ville.

Aux Etats-Unis particulièrement, on a consacré de nombreuses études à la recherche des mécanismes à travers lesquels se constitue le paysage urbain. Chapin se propose, dans la première partie de son ouvrage, de présenter une synthèse de ces travaux. C'est ainsi qu'il analyse les tentatives théoriques de Burgess, McKenzie, Hoyt, Harris et Ullman, Firey, etc., où l'on insiste tantôt sur les facteurs d'ordre économique, tantôt sur les facteurs d'ordre culturel. Mais cette démarche n'est, pour l'auteur, qu'un point de départ.

Son ambition est de contribuer à «l'élaboration d'un modèle théorique susceptible de guider le travail de l'urbaniste» (p. 5). Il est exact que l'urbaniste doit appuyer ses projets sur l'analyse scientifique de la réalité qu'il a dessein d'ordonner, que ses plans reflètent souvent davantage des conceptions personnelles que les voeux des citoyens eux-mêmes (p. 71). Il y a lieu toutefois, à notre sens, d'apporter quel- 
que nuance à la conclusion que Chapin tire de ces constatations. Il souhaite très justement la mise au point d'une théorie cohérente de l'aménagement de l'espace urbain, mais il faut bien voir l'insuffisance de cette démarche du point de vue de l'application: une doctrine de l'urbanisme reste nécessaire, puisque les enseignements de la science, s'ils doivent éclairer l'urbaniste, ne peuvent lui dicter toutes ses options. Le plan d'urbanisme est en définitive un projet politique et, à ce titre, il présuppose aussi un arbitrage conscient entre les aspirations divergentes des groupes sociaux.

La seconde partie de l'ouvrage constitue un très précieux recueil des principales techniques de recherche utilisées pour établir le «dossier urbain 》 nécessaire à l'élaboration du plan d'aménagement. Chapin décrit ainsi: les techniques d'étude de l'économie de la ville, en insistant sur celles qui permettent le mieux de situer cette dernière dans son contexte régional; les méthodes de prévision de l'emploi et de la population; les instruments d'analyse de l'utilisation du sol et de la circulation.

L'auteur expose, dans une troisième partie, le processus d'élaboration du «plan d'utilisation du sol »: il nous initie par là au travail même de l'urbaniste. Disposant du «dossier urbain 》 et aussi d'un arsenal de normes techniquement fondées, celui-ci a pour mission de proposer la ligne de conduite à suivre dans la répartition sur le territoire des divers éléments de la structure urbaine (usines, édifices commerciaux, résidences, etc.).

On ne saurait recommander l'ouvrage dє. Chapin comme une oeuvre de vulgarisation, mais l'urbaniste et le chercheur appelé à collaborer avec lui y verront un excellent instrument de travail.

\section{Yves Martin}

New Concepts in Wage Determination, édité par C.W. Taylor et F.C. Pierson, N.Y.: McGraw-Hill, 1957. 336 pp.

Ce livre est le fruit des réflexions de douze économistes qui ont participé de façon active, à un moment ou l'autre de leur carrière, à l'élaboration et à la réalisation de politiques de salaire.
L'unité du volume a été assurée par une série de rencontres, s'étendant sur une période de trois ans, au cours desquelles les auteurs ont développé un cadre commun d'analyse.

On retrouve, dans sept essais originaux, une appréciation de l'état actuel de la théorie des salaires et une tentative d'approche nouvelle à la lumière des développements présents. Le livre est, de plus, l'occasion d'une réinterprétation de l'économique du travail. On y modifie les vues traditionnelles en y intégrant des inquiétudes d'ordre institutionnel. L'étude des conditions du marché - c'est la thèse des auteurs - ne suffit plus à expliquer la formation des taux de salaire: il y faut ajouter une étude du conditionnement institutionnel. Un des buts du volume est de développer ce dernier concept en précisant l'influence des coutumes sociales, des attitudes communautaires, du statut des unions et des interventions gouvernementales sur la détermination des salaires. On accorde une importance particulière au rôle social de la grande entreprise, à l'agrandissement des marchés, aux effets de l'évaluation des tâches. On reproche à la théorie traditionnelle de ne pas accorder suffisamment d'importance aux pressions sociales dans la détermination des salaires: elle ne fait que les noter au point de départ pour n'en plus tenir compte ensuite au cours de l'analyse. Mais, disent les auteurs, dans l'esprit même des entrepreneurs, le prix de la marchandise ou le taux de profit a, dans la détermination des salaires, souventes fois moins d'importance que certaines pressions sociales.

C'est dans cette optique, élaborée dans les quatre chapitres de la première partie du volume, que les auteurs abordent dans une deuxième partie l'analyse de la structure des salaires et dans une troisième partie celle du niveau général des salaires.

Dunlop suggère un aspect «interne » et 《externe » à la structure des salaires, par référence à celle qui existe à l'intérieur d'une entreprise et à celle qui existe sur le plan soit de l'industrie ou d'une économie nationale. Lester tente d'analyser les réactions de la gérance à des changements dans la structures des salajres.

Dans la dernière partie, Reynolds et Kerr s'efforcent de démêler les influen- 